\title{
Photon Correlation Spectroscopy
}

National Cancer Institute

\section{Source}

National Cancer Institute. Photon Correlation Spectroscopy. NCI Thesaurus. Code C62329.

A type of spectroscopy that utilizes a laser beam to irradiate a sample containing particles in suspension, resulting in light scattering. Rapid fluctuations in scattering intensity, around a mean value at a certain angle, occur because of particle diffusion and are dependent upon on particle size. The calculated correlation function yields a diffusion coefficient, for a given temperature and viscosity, which can be used to calculate particle size. 